\title{
Abstract zu: Wer liest was? Die Konstruktion des männlichen Lesers in der Gegenwartsliteratur
}

Erschienen in: Unterstellte Leseschaften

Von: Simon Sahner

Literatur wird diskursiv in Kategorien eingeteilt. Dabei stellen die Kategorien von kanonisierter Hochliteratur und einer vermeintlich darunter stehenden sogenannten Unterhaltungs- und Trivialliteratur nur die beiden sichtbarsten dar. Vielmehr versammeln sich unter der Frage, wer welche literarischen Texte vermeintlich liest, zahlreiche Zuordnungen zu Kategorien, die ein weitaus differenzierteres Feld darstellen. Die Frage nach den Leser*innen ist sowohl in der empirisch-analytisch arbeitenden Literatursoziologie als auch in der eher hermeneutisch analysierenden Literaturwissenschaft wiederholt gestellt und bearbeitet worden. Während die Literatursoziologie teilweise unter Erhebung empirischer Daten das Rezeptionsverhalten von tatsächlichen Leser*innen, den Rezipient*innen, analysiert, hat sich die Literaturwissenschaft vorrangig Konstrukten von Leser*innen zugewandt, die statt der Rezipient*innen die Adressat*innen eines Textes in den Blick nehmen und die Frage stellen, welcher Leser*innentypus strukturell und thematisch angesprochen wird. Hier sind vor allem Theoriekonstrukte wie Wolfgang Isers impliziter Leser oder Erwin Wolffs intendierter Leser zu nennen, die sich mit methodischen Unterschieden alle auf Leser*innen als potenzielle, ideale Rezeptionsinstanzen eines Textes beziehen. Es zeigt sich aber, dass es in der diskursiven Aushandlung von literarischen Texten und ihrer Kontextualisierung im Verlauf dieses Prozesses zu Kategorisierungen kommt, die sowohl durch textuelle, durch paratextuelle und nicht zuletzt auch durch ökonomische Einflüsse zustande kommen. Zu einer Analyse dieser Zuordnungsprozesse bedarf es eines Vorgehens, das sich nicht auf eine der beiden Methoden aus Literatursoziologie und -wissenschaft beschränkt.

Unter Verwendung von Methoden aus beiden Wissenschaftsfeldern setzt sich der Beitrag mit den Kategorien der Männer- und der Frauenliteratur auseinander. Anders als der Begriff der Männerliteratur weist Frauenliteratur eine weitzurückreichende Historie als vor allem abwertender Terminus zur Abgrenzung gegenüber einer unmarkierten eigentlichen Literatur auf. Dabei zeigt sich bei genauerer Betrachtung von Aspekten der impliziten Leser*innenkonstruktion, der paratextuellen Adressat*innenansprache und dem diskursiven Umgang mit vielen unmarkierten literarischen Texten, dass sie vielmehr ein komplementäres Gegenüber zur sogenannten Frauenliteratur darstellen bzw. selbst in zusätzliche, häufig nicht weiter definierte Bereiche eingeordnet werden. Beispielhaft 
wird an dem Roman Die jüngsten Tage von Tom Müller dargelegt, wie er sich in den genannten Aspekten als Männerliteratur positioniert und einen männlich assoziierten impliziten Leser konstruiert, der aber nicht allein in der Textstruktur zu erkennen ist, sondern den Roman diskursiv umspannt. Als Erkenntnis steht am Ende, dass es einer detaillierten Betrachtung solcher Zuordnungen bedarf, um strukturelle Ungleichheiten zu erkennen, die sich nicht allein auf die Kategorien Frauenliteratur und eigentliche Literatur beschränken.

Die Langfassung dieses Beitrages in der Reihe „Unterstellte Leseschaften" finden Sie $\underline{\text { hier. }}$.

SUGGESTED CITATION: Sahner, Simon: Abstract zu: Wer liest was? Die Konstruktion des männlichen Lesers in der Gegenwartsliteratur, in: KWI-BLOG, [https://blog.kulturwissenschaften.de/abstract-zu-wer-liest-was/], 03.05.2021

DOI: https://doi.org/10.37189/kwi-blog/20210503-0831

DuEPublico

Duisburg-Essen Publications online

\section{DEUISEBN R G}

offen im Denken

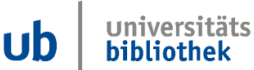

Dieser Text wird via DuEPublico, dem Dokumenten- und Publikationsserver der Universität Duisburg-Essen, zur Verfügung gestellt. Die hier veröffentlichte Version der E-Publikation kann von einer eventuell ebenfalls veröffentlichten Verlagsversion abweichen.

DOI: $\quad$ 10.37189/kwi-blog/20210503-0831

URN: urn:nbn:de:hbz:464-20210503-164537-0 